\title{
EPR STUDY OF FREE RADICALS FORMED IN FUSIDIC ACID AND NEOMYCIN UNDER UV IRRADIATION
}

\author{
EWA PIERZCHAŁA ${ }^{1}$, PAWEŁ RAMOS ${ }^{2 *}$ and BARBARA PILAWA ${ }^{2}$
}

${ }^{1,2}$ School of Pharmacy with the Division of Laboratory Medicine in Sosnowiec, Medical University of Silesia in Katowice, Poland

${ }^{1}$ Department of Esthetical Medicine, Francuska 20-24, 40-022 Katowice, Poland, ${ }^{2}$ Department of Biophysics, Jedności 8, 41-200 Sosnowiec, Poland

\begin{abstract}
Free radicals in UV irradiated antibiotics used in dermatology were examined. Concentrations of free radicals in fusidic acid and neomycin in ointment were determined. EPR spectra of the tested antibiotics were measured by electron paramagnetic resonance spectrometer with magnetic modulation of $100 \mathrm{kHz}$ and numerical acquisition system the Rapid Scan Unit. The influence of microwave powers in the range of 2.2-70 $\mathrm{mW}$ on the spectra was obtained. Amplitudes $(\mathrm{A})$ and linewidths $\left(\Delta \mathrm{B}_{\mathrm{pp}}\right)$ of the EPR spectra, were analyzed. The EPR spectra were homogeneously broadened. Fast spin-lattice relaxation processes existed in UV irradiated fusidic acid and neomycin, which EPR spectra were not saturated up to $70 \mathrm{~mW}$. The influence of the time of UV irradiation on free radicals in the samples was observed. The samples were irradiated by UVA $(315-400 \mathrm{~nm})$ in the 30, 60, and $90 \mathrm{~min}$ period. Free radical concentrations in the tested antibiotics exposed to UV were proportional to the amplitudes (A) of the EPR spectra. The highest amplitudes (A) were observed for the UV irradiated antibiotics during for $60 \mathrm{~min}$. Fusidic acid was characterized by higher amplitudes (A) than neomycin. Fusidic acid and neomycin in ointment used to treat bacterial infection of skin under UV irradiation may produce free radical toxic effects. The stronger photosensitivity characterized fusidic acid relatively to neomycin. EPR spectroscopy is a useful method of testing free radicals formed in antibiotics during photolysis.
\end{abstract}

Keywords: UV irradiation, free radicals, EPR spectroscopy, fusidic acid, neomycin, dermatology

The two ointments containing fusidic acid or neomycin as the strong antibiotics used in dermatology were examined in terms of free radical formation during UV irradiation. UV electromagnetic waves produce free radicals via photolysis processes in drugs (1-2), skin (3-4), and melanins (5-7). Unpaired electrons are responsible for high chemical activity of free radical molecules (8-9). Free radical reactions as toxic transformations in the skin are not expected during therapy $(3-4,10-12)$. The aim of this work was to check the hypothesis about the production of free radicals under UV irradiation of the tested therapeutic ointments with fusidic acid or neomycin. Free radical properties and amounts in the UV irradiated fusidic acid and neomycin in ointment samples were determined. The effect of UV irradiation time on free radicals in these antibiotics was evaluated.

Chemical structures of fusidic acid $\left(\mathrm{C}_{31} \mathrm{H}_{48} \mathrm{O}_{6}\right)$ and neomycin $\left(\mathrm{C}_{23} \mathrm{H}_{46} \mathrm{~N}_{6} \mathrm{O}_{13}\right)$, were shown in Figure 1a-b (13), respectively.
Fusidic acid is the natural antibiotic derived from Fusidium coccineum, Mucor ramannianus and Isaria kogana (14-16). Fusidic acid interacts with Staphylococcus aureus, $\beta$-hemolytic Streptococci, Enterococci, Corynebacterium, Gram-negative bacteria, Mycobacterium leprae (14-16). Neomycin is the aminoglycoside antibiotic, which highly interacts with Gram-negative bacteria and it partially interacts with Gram-positive bacteria (14-16). This antibiotic is highly effective in killing Enterobacter cloacae, Escherichia coli, and Proteus vulgaris (1416). Fusidic acid and neomycin are effective in treating skin infections (16).

Free radical formation in fusidic acid and neomycin, was not tested so far. In this study, the susceptibility of the chemical structure of these two antibiotics on UV irradiation was compared. The practical aspect of the performed analysis was to obtain information about the possibility of skin exposition on UV waves during treatment by fusidic acid or neomycin as the components of ointments.

\footnotetext{
* Corresponding author: e-mail: pawelramos@sum.edu.pl
} 


\section{EXPERIMENTAL}

\section{Samples}

Two antibiotics used in dermatology, such as fusidic acid and neomycin in an ointment, were examined. The antibiotics were components of ointments. The white vaseline was used as the bases. Nonirradiated and UV irradiated samples were tested in this study. These antibiotics were irradiated by UVA electromagnetic waves with wavelengths $(\lambda)$ in the range of 315-400 $\mathrm{nm}$ during four different times: 30, 60, and $90 \mathrm{~min}$. For UV irradiation the Medisun 250 lamp (Germany) with 4 radiators with power of $20 \mathrm{~W}$ was used. The antibiotic samples were situated as close as $30 \mathrm{~cm}$ from the lamp.

The samples were located in thin-walled glass tubes with the an external diameter of $1 \mathrm{~mm}$. The empty tubes were free of EPR signals. The masses of the antibiotic samples in the tubes were calculated as the difference of the masses of the tubes with the antibiotic and the masses of the empty tubes. The measurements were done by the weight of Sartorius Firm (Germany).

\section{EPR measurements}

Electron paramagnetic resonance measurements were performed by the use of an X-band $(9.3 \mathrm{GHz})$ EPR spectrometer with magnetic modulation of $100 \mathrm{kHz}$ produced by of Radiopan Firm (Poznan, Poland). The total microwave power produced by klystron was $70 \mathrm{~mW}$. Microwave power during the experiment was changed by application of different attenuations: $15-0 \mathrm{~dB}$, which approximated powers in the range $2.2-70 \mathrm{~mW}$. Microwave frequency and magnetic induction were measured by MCM101 recorder and NMR magnetometer of EPRAD Company (Poznań, Poland), respectively. Numerical acquisition of EPR spectra was done by the Rapid Scan Unit of Jagmar Company (Kraków, Poland) linked with the EPR spectrometer. The measuring time of the individual EPR line was 1 second. Because of the noise and the low amplitudes, the EPR spectra were accumulated 50 times. Amplitudes (A) were divided by the mass of the whole ointment sample located in the glass tubes. The mass of the individual sample was calculated as the difference between the mass of the tube containing the ointment and the mass of the empty tube. Measurement for the tested ointment samples was replicated three times. New tested samples were prepared. Therefore, they were irradiated and EPR measured three times. The results of amplitudes and linewidths are the average of three measurements.

\section{EPR analysis}

The amplitudes (A), integral intensities (I), linewidths $\left(\Delta \mathrm{B}_{\mathrm{pp}}\right)$, and $\mathrm{g}$-factors of the first-derivative EPR spectra were analyzed. The parameters of the EPR spectra of the tested UV irradiated samples recorded with the low microwave power of $2.2 \mathrm{~mW}$ without microwave saturation effects, were compared. The influence of microwave power on amplitudes $(\mathrm{A})$ and linewidths $\left(\Delta \mathrm{B}_{\mathrm{pp}}\right)$ of the spectra were determined. g-Factor was calculated from the reso-

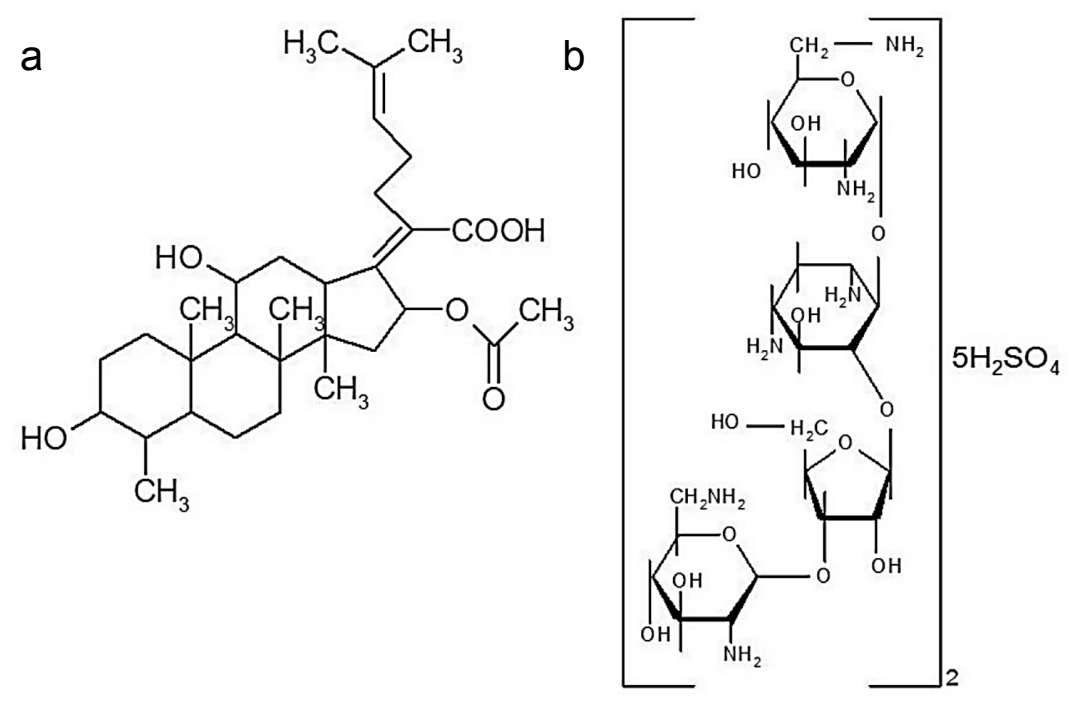

Figure 1. Chemical structure of (a) fusidic acid and (b) neomycin (13) 
a

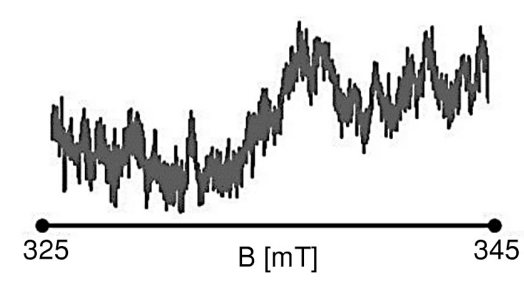

b

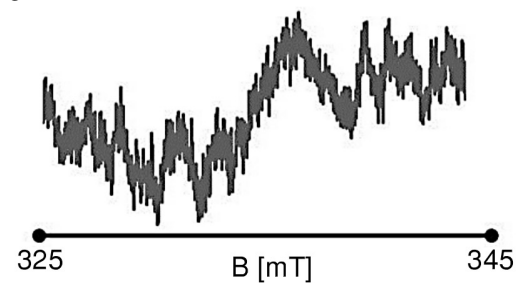

Figure 2. EPR spectra of (a) fusidic acid and (b) neomycin in ointment after UV irradiation by 60 min. The measurements were performed with a microwave power of $2.2 \mathrm{~mW}$. B is the magnetic induction of the field produced bythe the electromagnet

nance condition as (17-20): $\mathrm{g}=\mathrm{h} v / \mathrm{\mu}_{\mathrm{B}} \mathrm{B}_{\mathrm{r}}$, where: $\mathrm{h}-$ Planck constant, $v-$ microwave frequency, $\mu_{B}-$ Bohr magneton, $\mathrm{B}_{\mathrm{r}}$ - induction of resonance magnetic field. Free radical concentrations $(\mathrm{N})$ in the UV irradiated antibiotics were proportional to the amplitudes (A) of the EPR spectra measured with the low microwave power of $2.2 \mathrm{~mW}$.

EPR spectra were measured and analyzed by the professional spectroscopic programs of Jagmar Company (Kraków, Poland) and LabVIEW 8.5 of National Instruments Company (Texas, USA). The Origin (USA) and Microsoft Excel (USA) programs were used.

\section{RESULTS AND DISCUSSION}

Fusidic acid, neomycin, white vaseline, and the ointments containing fusidic acid and neomycin, before UV irradiation were diamagnetic without unpaired electrons in molecules. Diamagnetic character of the antibiotic samples resulted from chemical structure, which was shown in Figure 1a-b (13). All the tested samples with the zero magnetic moment did not absorb microwaves in the magnetic field and the EPR signals were not obtained even at a high microwave power of $70 \mathrm{~mW}$. The tested samples revealed high purity. Paramagnetic improvements were not found in their structure.

In this work, free radicals the UV irradiated samples, composed of the antibiotic and white vaseline as the base were examined and all the presented data related to these complex samples. Different free radical formation in the pure samples of fusidic acid, neomycin and vaseline exposed to UV irradiation was expected. The reactions between free radicals of the antibiotic components and vaseline base may effect on the resultant free radical contents in the complex samples of the ointments. In the clinical practice, the ointments containing the pharmacologically active substance and the base are used.
Therefore, the research results obtained by using EPR spectroscopy were helpful to indicate the storage conditions of the ointments. The information about free radicals in the real samples exposed to UV was obtained.

After UV irradiation both the diamagnetic antibiotics: fusidic acid and neomycin in ointments become paramagnetic. Photolysis produced paramagnetic centers in both tested antibiotics. The apparent g-values of the EPR spectra resulted from all types of free radicals formed by UV irradiation in the ointments were obtained near 2. Because of the high noise in the experimental spectra, only the apparent g-factors were obtained. Taking into account the chemical structures of fusidic acid (Fig. 1a) (13) and neomycin (Fig. 1b) (13), it can be said that unpaired electrons in free radicals of these two tested substances are mainly located on carbon and oxygen atoms. Additionally, the unpaired electrons located on nitrogen atoms may be expected in UV irradiated neomycin. The measured EPR spectra were the sum of lines of all types of free radicals existed in the tested ointments. Because of the high level of noise, deconvolution of the spectra was not done and the individual component lines were not obtained. The exemplary EPR spectra of UV irradiated fusidic acid and neomycin in ointments were shown in Figure 2a-b, respectively. The time of UV irradiation was $60 \mathrm{~min}$. The spectra in Figure 2a-b were not saturated, because of the low microwave power $(2.2 \mathrm{~mW})$ used during the measurements. Free radicals in drugs were also formed by thermolysis (21-24). EPR spectra were measured for the heated drugs. Photolysis of the ointments containing the antibiotic and vaseline was accompanied by the weaker formation of free radicals than thermolysis. The lower EPR lines were measured for the products of photolysis tested in this work than the products of thermolysis (25). Only the noisy EPR lines with the very low amplitudes (Fig. 2) were measured for the 

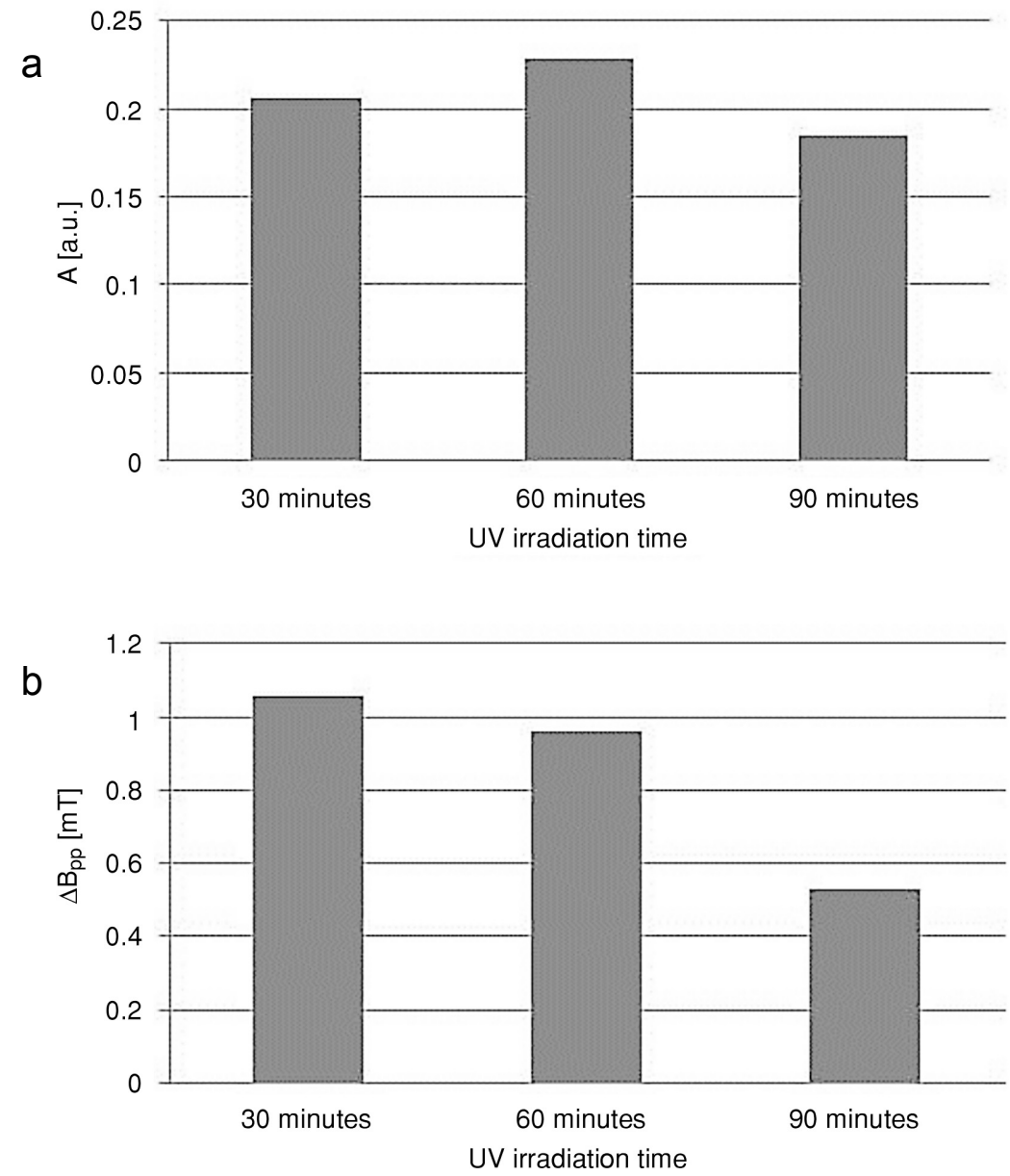

Figure 3. The effect of the time of UV irradiation on (a) amplitudes (A) $\left[ \pm 0.01\right.$ a. u.] and (b) linewidths $\left(\Delta \mathrm{B}_{\mathrm{pp}}\right)[ \pm 0.04 \mathrm{mT}]$ of the EPR spectra of fusidic acid in a ointment for the measurements with the low microwave power of $2.2 \mathrm{~mW}$

UV irradiated ointments tested in this work. The higher EPR lines were obtained for these ointments after thermal treatment, which was described in our earlier work (25). Unfortunately, the values of integral intensities (I) of the ointments containing fusidic acid and neomycin after UV irradiation and after thermal treatment, were not compared in the present paper. Because of the high level of noise, the integral intensities (I) were not calculated for the low EPR signals of the ointments exposed to UV. However, it can be said that the EPR lines of the present examined ointments stored under UV were considerably lower than EPR lines of these samples, which were stored earlier at the higher temperatures (up to $50^{\circ} \mathrm{C}$ ) $(25)$.

The parameters of the EPR spectra of the examined antibiotics depended on the time of UV irradiation. The amplitudes (A) of the EPR spectra of fusidic acid and neomycin in ointments exposed to UV during 30, 60, and 90 min were compared in Figures $3 \mathrm{a}$ and $4 \mathrm{a}$, respectively. The amplitude (A) of EPR lines of fusidic acid in ointments increased with irradiation time (Fig. 3a), and for neomycin in ointments, the highest amplitude (A) was observed for $60 \mathrm{~min}$ of irradiation (Fig. 4a). The changes of free radical amounts in the samples were responsible for the changes of amplitudes (A) of EPR lines. The amplitudes (A) of the EPR lines of the tested ointments decreased after 90 min of exposition on UV related to the $60 \mathrm{~min}$ of irradiation (Figs. $3 \mathrm{a}$ and $4 \mathrm{a}$ ). Probably free radical recombination or interactions of free radicals with oxygen may be responsible for this effect.

Linewidths $\left(\Delta \mathrm{B}_{\mathrm{pp}}\right)$ of the EPR spectra of fusidic acid and neomycin in ointments exposed to UV during 30, 60, and 90 min were compared in Figures $3 \mathrm{~b}$ and $4 b$, respectively. The highest value of linewidth $\left(\Delta \mathrm{B}_{\mathrm{pp}}\right)$ of the EPR spectra of fusidic acid in oint- 
ments was visible after $30 \mathrm{~min}$ of UV irradiation (Fig. 3b). The highest linewidth $\left(\Delta \mathrm{B}_{\mathrm{pp}}\right)$ of the EPR spectra of neomycin in ointments was obtained after 90 min of UV irradiation (Fig. 4b). The narrowest EPR lines characterizing fusidic acid in ointments irradiated for $90 \mathrm{~min}$ (Fig. 3b) and neomycin in ointments irradiated for $60 \mathrm{~min}$ (Fig. 4b). Linewidths $\left(\Delta \mathrm{B}_{\mathrm{pp}}\right)$ depended on magnetic interactions between unpaired electrons of free radicals in the UV irradiated antibiotics. The enhancement of dipolar interactions increased linewidths (19-20).

The EPR lines of UV irradiated fusidic acid and neomycin in ointments were not saturated in the adjusted level of microwave power $(2.2-70 \mathrm{~mW})$. The changes of amplitudes (A) the EPR lines of fusidic acid and neomycin in ointments exposed to UV under 30, 60 and $90 \mathrm{~min}$, were presented in (Figs. 5a-c and 6a-c), respectively. The amplitudes
(A) increased while microwave power was increasing. The correlations in Figures 5a-c and 6a-c indicated that fast spin-lattices existed in the tested antibiotics, regardless of irradiation time.

The EPR spectra of UV irradiated fusidic acid and neomycin in ointments were homogenously broadened. Typical correlations between the spectral parameters and microwave power were obtained (Figs. 7a-c and 8a-c). Linewidths $\left(\Delta \mathrm{B}_{\mathrm{pp}}\right)$ of the EPR spectra of fusidic acid and neomycin in ointments irradiated during 30, 60 and 90 min increased with enhancing of microwave power.

Free radical concentrations in fusidic acid and neomycin in ointments as the value proportional to the spectral amplitude (A) depended on UV irradiation time. The values of amplitudes were shown in diagrams in Figures $3 \mathrm{a}$ and $4 \mathrm{a}$, respectively. The highest free radical concentration occurred in fusidic
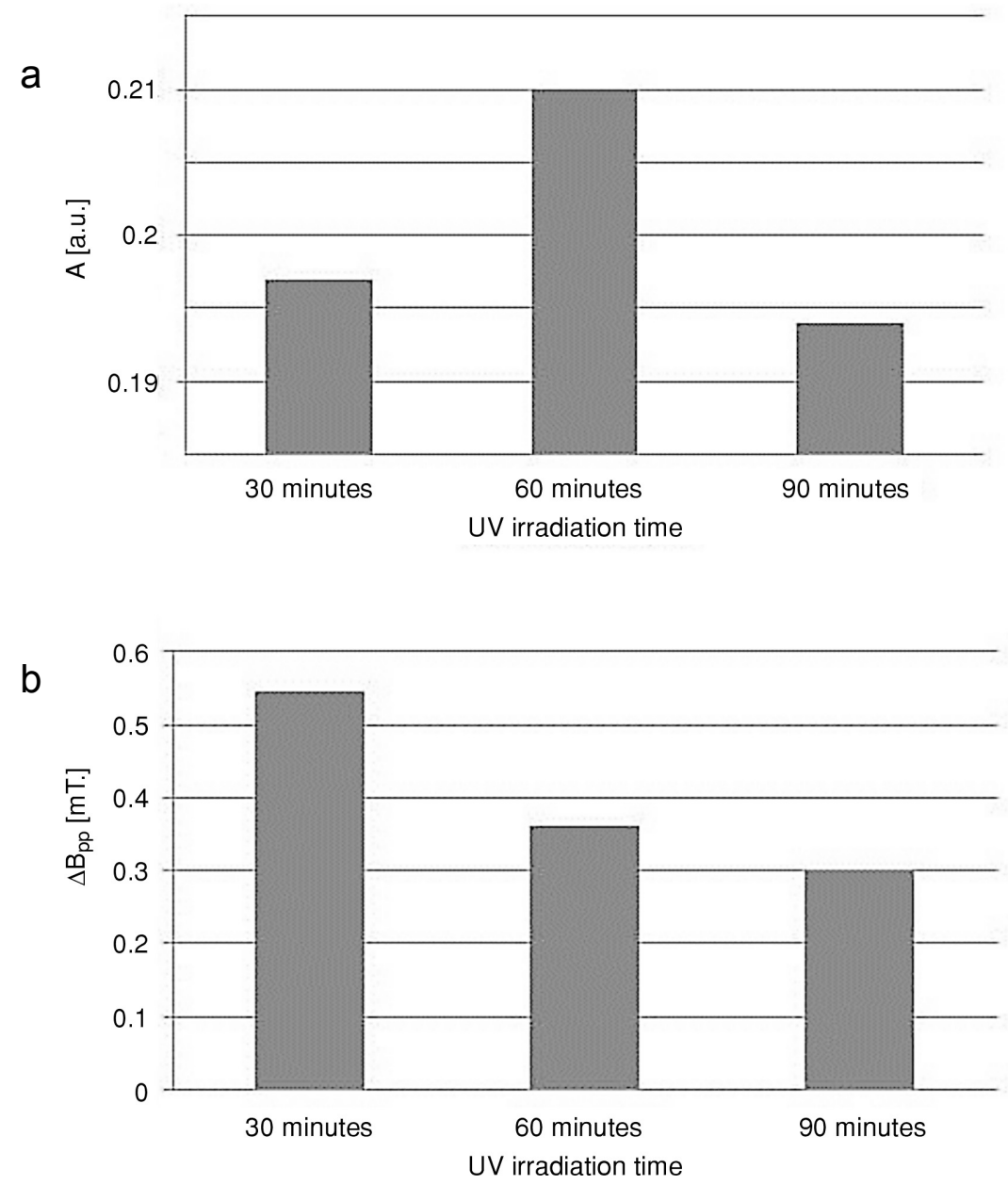

Figure 4. The effect of the time of UV irradiation on (a) amplitudes (A) $\left[ \pm 0.01\right.$ a. u.] and (b) linewidths $\left(\Delta \mathrm{B}_{\mathrm{pp}}\right)[ \pm 0.04 \mathrm{mT}]$ of the EPR spectra of neomycin in ointment for the measurements with low microwave power of $2.2 \mathrm{~mW}$ 
acid exposed to UV during 60 min (Fig. 3a). The lowest free radical concentration was fusidic acid UV irradiated by 90 min (Fig. 3a). The highest and the lowest free radical concentrations in neomycin were obtained for the samples irradiated by 60 and 90 min (Fig. 4a).

Additionally, the effect of UV irradiation during $60 \mathrm{~min}$ on the pure neomycin, fusidic acid, and
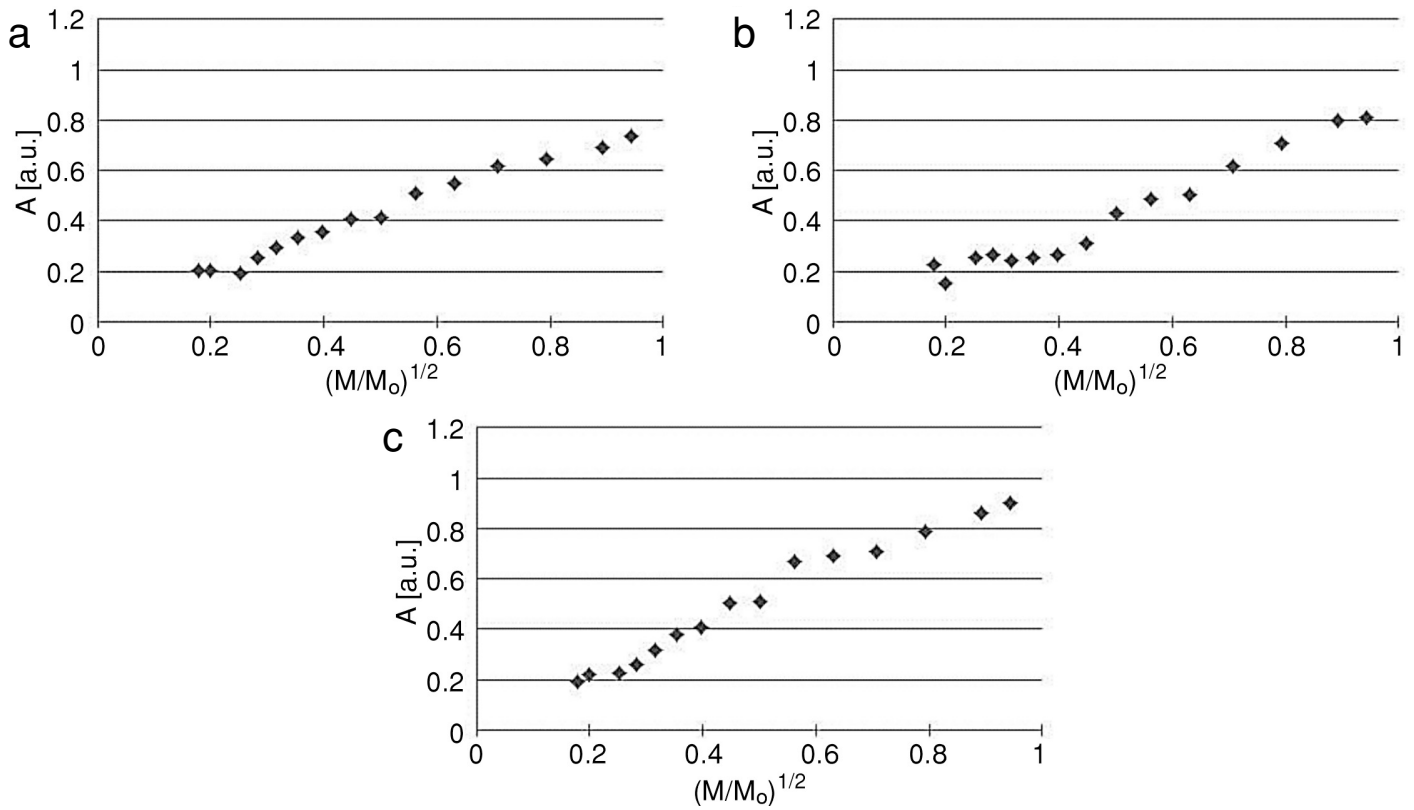

Figure 5. The changes of the amplitudes (A) $[ \pm 0.01$ a. u.] of EPR spectra of UV irradiated fusidic acid in the ointment with microwave power $\left(\mathrm{M} / \mathrm{M}_{\mathrm{o}}\right)$. The correlations for fusidic acid in ointment irradiated during: (a) $30 \mathrm{~min}$, (b) $60 \mathrm{~min}$, and (c) $90 \mathrm{~min}$. $\mathrm{M}, \mathrm{M}_{\mathrm{o}}-\mathrm{microwave}$ power used during the measurement, and the total microwave power produced by klystron $(70 \mathrm{~mW})$, respectively
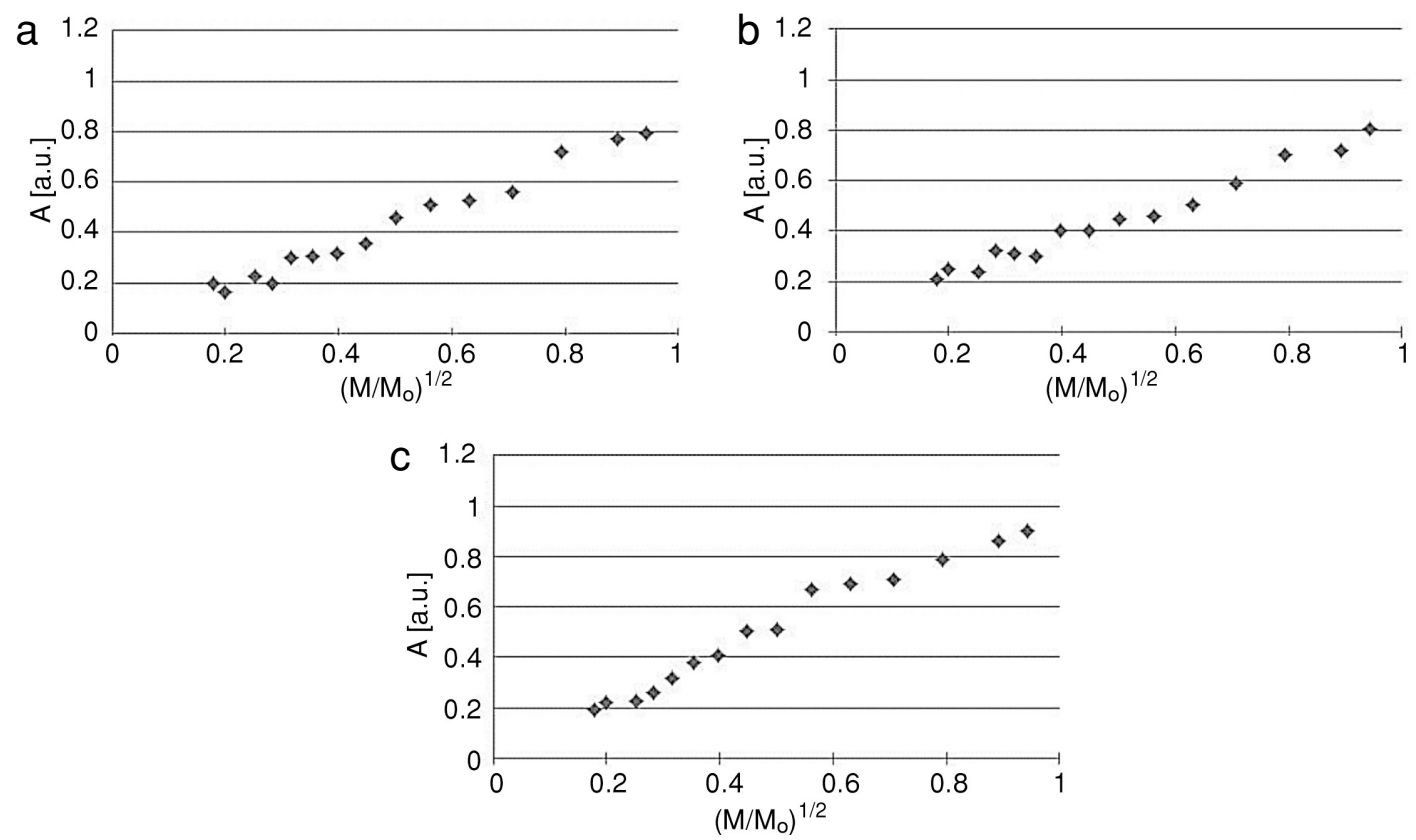

Figure 6. The changes of the amplitudes (A) $[ \pm 0.01$ a. u.] of EPR spectra of UV irradiated neomycin in the ointment with microwave power $\left(\mathrm{M} / \mathrm{M}_{\mathrm{o}}\right.$ ). The correlations for neomycin in ointment irradiated during: (a) $30 \mathrm{~min}$, (b) $60 \mathrm{~min}$, and (c) $90 \mathrm{~min}$. M, $\mathrm{M}_{\mathrm{o}}-\mathrm{microwave}$ power used during the measurement, and the total microwave power produced by klystron $(70 \mathrm{~mW})$, respectively 

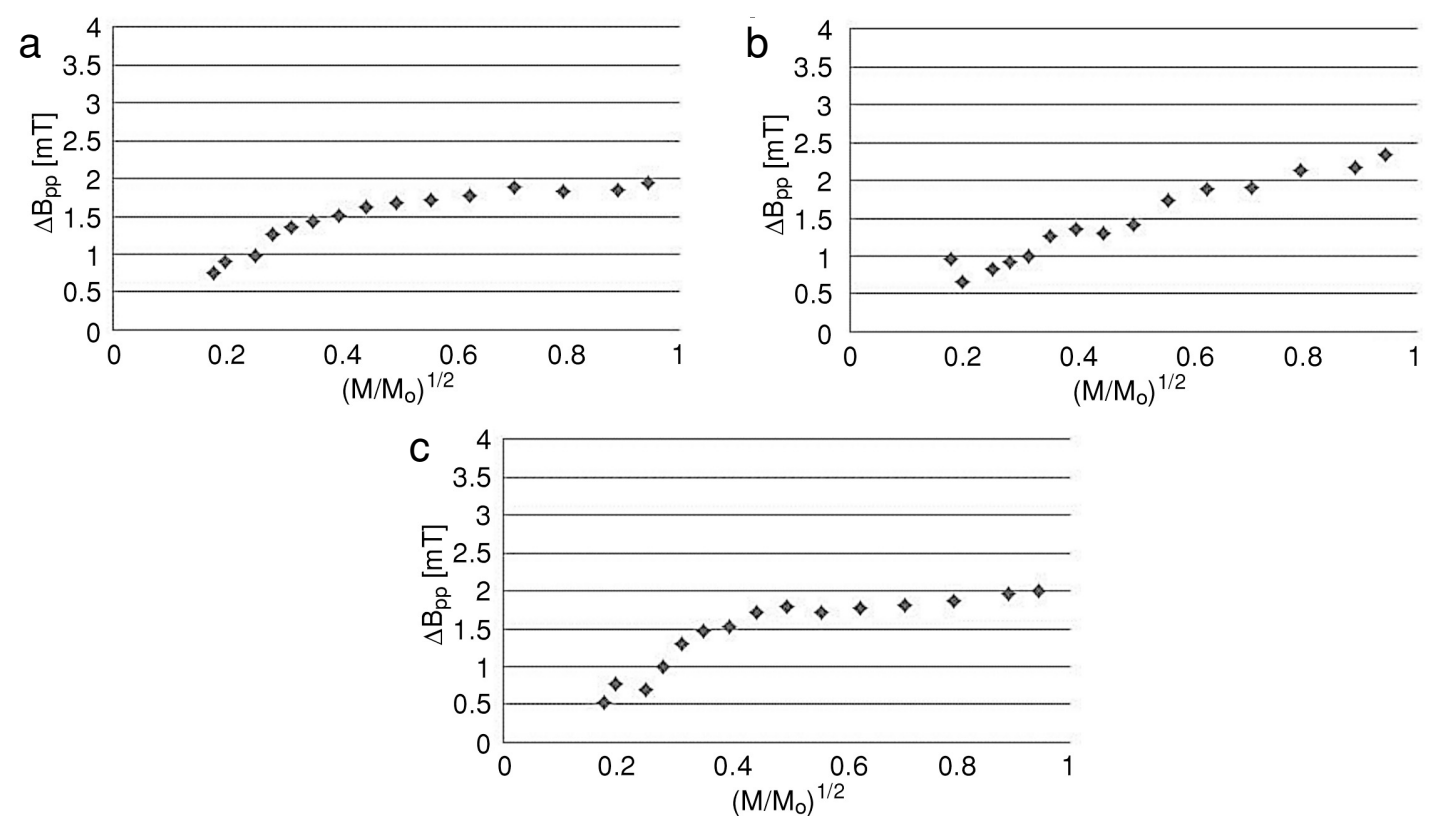

Figure 7. The changes of the linewidths $\left(\Delta \mathrm{B}_{\mathrm{pp}}\right)[ \pm 0.04 \mathrm{mT}]$ of EPR spectra of UV irradiated fusidic acid in ointment with microwave power $\left(\mathrm{M} / \mathrm{M}_{\mathrm{o}}\right.$ ). The correlations for fusidic acid in ointment irradiated during: (a) $30 \mathrm{~min}$, (b) $60 \mathrm{~min}$, and (c) $90 \mathrm{~min}$. M, $\mathrm{M}_{\mathrm{o}}-\mathrm{microwave}$ power used during the measurement, and the total microwave power produced by klystron $(70 \mathrm{~mW})$, respectively
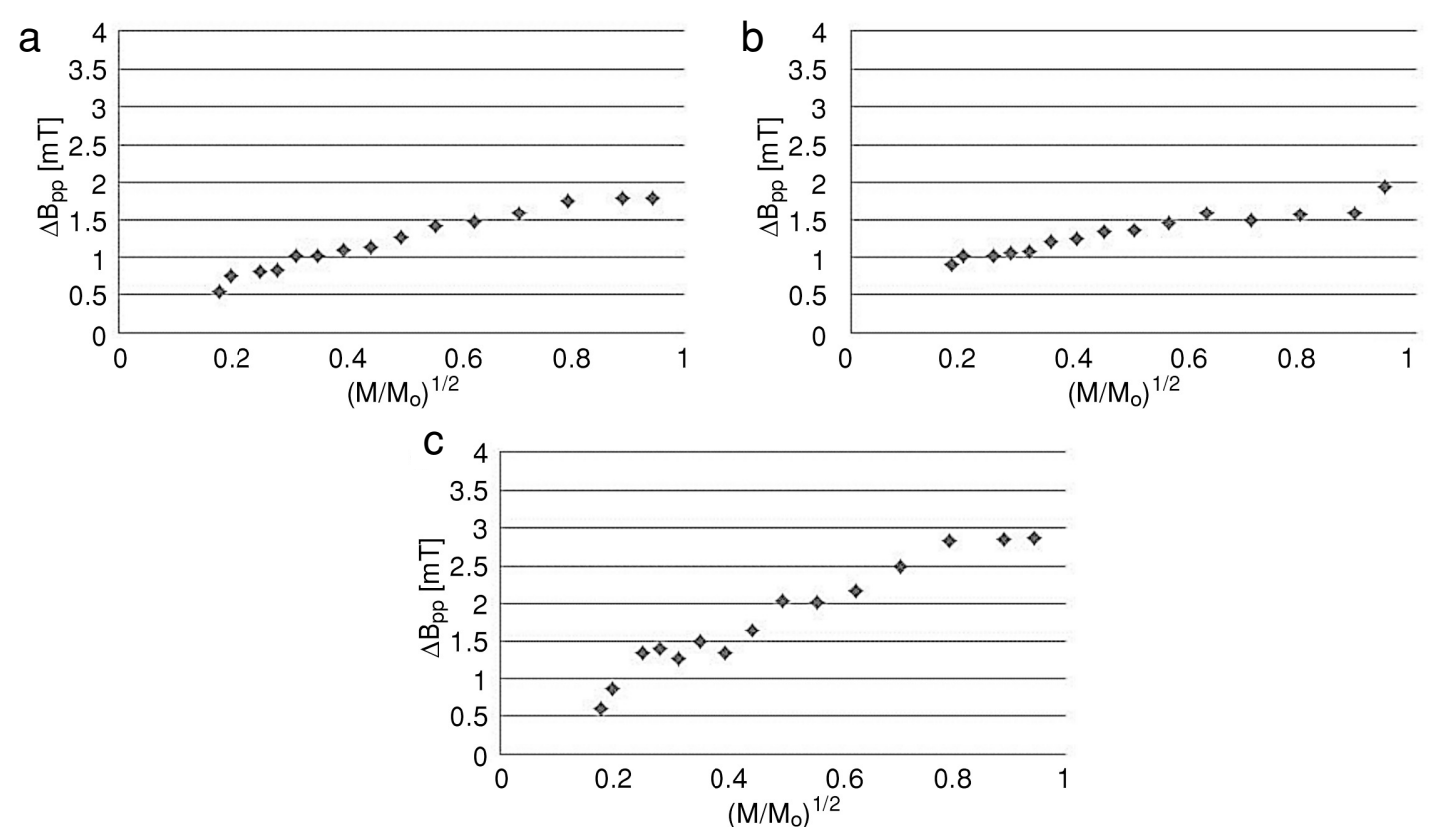

Figure 8 . The changes of the linewidths $\left(\Delta \mathrm{B}_{\mathrm{pp}}\right)[+0.04 \mathrm{mT}]$ of EPR spectra of UV irradiated neomycin in ointment with microwave power $\left(\mathrm{M} / \mathrm{M}_{\mathrm{o}}\right.$ ). The correlations for neomycin in ointment irradiated during: (a) $30 \mathrm{~min}$, (b) $60 \mathrm{~min}$, and (c) $90 \mathrm{~min}$. M, $\mathrm{M}_{\mathrm{o}}-\mathrm{microwave}$ power used during the measurement, and the total microwave power produced by klystron $(70 \mathrm{~mW})$, respectively

white vaseline, was studied. The EPR spectra of these two antibiotics and the vaseline base were shown in Figure 9. Under UV free radicals were formed in all the components of the ointments, in both the antibiotics and the base. The highest EPR signal was observed for UV irradiated vaseline (Fig. 


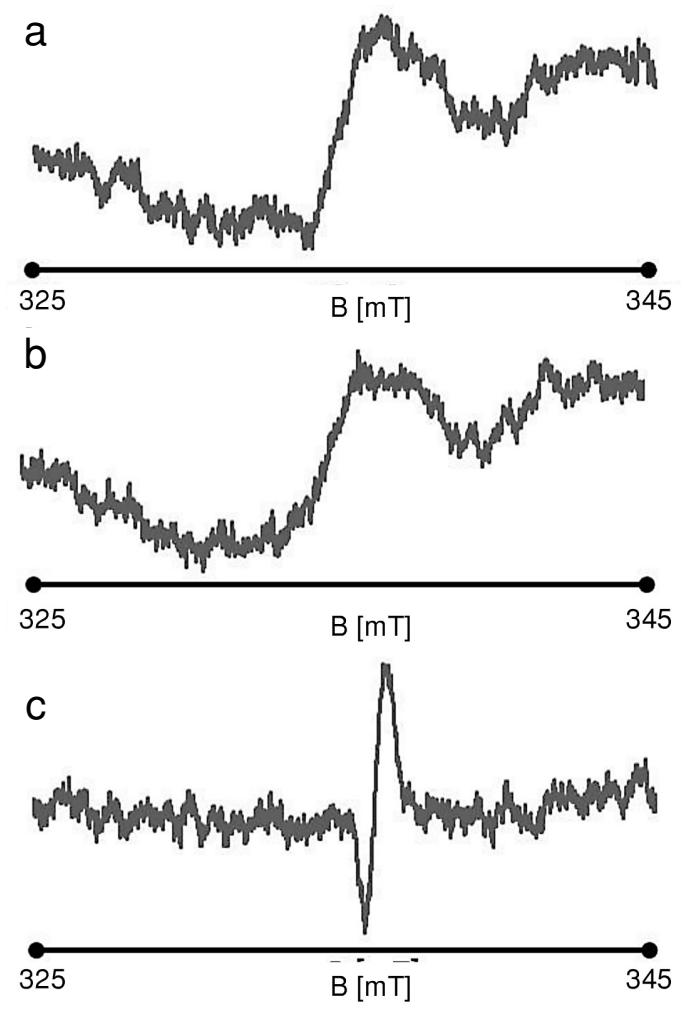

Figure 9. EPR spectra of (a) fusidic acid, (b) neomycin and (c) white vaseline after UV irradiation by $60 \mathrm{~min}$. The measurements were performed with a microwave power of $2.2 \mathrm{~mW}$. B is the magnetic induction of the field produced bythe electromagnet

9c). The base of the studied ointment contained the highest amounts of free radicals. It indicated that the main samples studied in this work as the ointment containing both the antibiotic and the vaseline base, revealed EPR lines come from the active ant the neutral part of the ointment.

Electron paramagnetic resonance (EPR) spectroscopy with a microwave frequency of $9.3 \mathrm{GHz}$ is the useful method of examining free radicals formed in antibiotics for dermatology. This spectroscopic method gives information about types, concentrations, and properties of free radicals formed in drugs by photolysis. Photolysis processes may appear during therapy or storage of antibiotics.

\section{CONCLUSIONS}

EPR studies of the influence of UV irradiation on the two tested antibiotics applied in dermatology proved that ultraviolet waves changed diamagnetic to paramagnetic character of fusidic acid and neomycin. For the tested samples EPR lines appeared. The EPR lines were homogeneously broadened. The EPR lines were not saturated in the used range of microwave power $(2.2-70 \mathrm{~mW})$, so the fast spin-lattice relaxation existed in UV irradiated fusidic acid and neomycin. Free radicals were formed under UV irradiation in fusidic acid and neomycin. Fusidic acid was characterized by higher free radical concentration than neomycin. Fusidic acid used to treat bacterial infection of the skin may produce high free radical toxic effects during therapy. Free radical concentration enhanced with increasing of UV irradiation time in both tested antibiotic samples. The stronger photosensitivity was observed for fusidic acid than for neomycin.

\section{Ackonowledgments}

This study was financially supported by Medical University of Silesia in Katowice, the grant no. KNW-1-059/K/7/O

\section{REFERENCES}

1. Ramos P., Pilawa B.: Med. Chem. Res. 23, 8 (2014).

2. Dołowy M., Ramos P., Pilawa B.: Int. J. Photoenergy, 2014, ID 953619 (2014).

3. Hanson K. M., Gratton E., Bardeen Ch.: J. Free Radic. Boil. Med. 41 (2006).

4. Jurkovic P., Sentjurc M., Gasperlin M., Kristl J., Pecar S.: Eur. J. Pharm. Biopharm. 56, (2003).

5. Zdybel M., Chodurek E., Pilawa B.: J. Appl. Biomed. 13, 2 (2015).

6. Zdybel M., Pilawa B.: Nukleonika 60, 3 (2015).

7. Beberok A., Zdybel M., Pilawa B., Buszman E., Wrześniok D.: Chem. Phys. Lett. 592, 1 (2014).

8. Halliwell B.:J. Free Radic. Biol. med. 46, 5 (2009).

9. Moskovitz J., Yim M. B., Chock P. B.: Arch. Biochem. Biophys. 397, 2 (2002).

10. Poljsak B., Dahmane R.: Derm. Res. Pract. 2012, ID 135206 (2012).

11. Pham-Huy L. A., He H., Pham-Huyc Ch.: Intern. J. Biomed. Sci. 4, 2 (2008).

12. Bartosz G.: The second face of oxygen. Free radicals in nature. PWN, Warsaw 2006 (in Polish).

13. Zejca A., Gorczyca M.: Medicinal Chemistry. PZWL, Warsaw 2004 (in Polish).

14. Kostowski W., Herman S. Z.; Pharmacology. PZWL, Warsaw 2007 (in Polish).

15. Janiec W., Krupińska J.: Pharmacodynamics. PZWL, Warsaw1999 (in Polish). 
16. Janicki S., Fiebig A.: Applied Pharmacy. PZWL, Warsaw 1998 (in Polish).

17. Eaton G. R., Eaton S. S., Salikhov K. M.: Foundations of Modern EPR. World Scientific, Singapore, New Jersey, London, Hong Kong 1998.

18. Weil J.A, Bolton J. R.: Electron Paramagnetic Resonance: Elementary Theory and Practical Applications, 2nd edn. John Wiley \& Sons, New York 2007.

19. Stankowski J., Hilczer W.: Introduction to magnetic resonance spectroscopy. PWN, Warsaw 2005 (in Polish).
20. Wertz J. E., Bolton J.R.: Electron Spin Resonance: Elementary Theory and Practical Applications. Chapman and Hall, London 1986.

21. Kościelniak-Ziemniak M., Pilawa B.: Appl. Magn. Res. 42, 4 (2012).

22. Krztoń A., Liszka B., Ramos P., Pilawa B.: Eng. Biomater. 12, 89 (2009).

23. Ramos P., Pilawa B., Stroka E.: Nukleonika 58, 3 (2013).

24. Ramos P., Pilawa B.: Bioinorg. Chem. Applicat. 2014, ID 547032 (2014).

25. Pierzchała E., Ramos P., Pilawa B.: Acta Pol. Pharm. 74, 4 (2017).

Received: 26.01.2018 